\title{
Adapting the modified barium swallow: modifications to improve safety in the setting of airborne respiratory illnesses like COVID-19
}

\author{
Alice R. Goldman ${ }^{1}$ (D) Jay K. Pahade ${ }^{2}$. Nicole A. Langton-Frost ${ }^{3} \cdot$ Chiquacta A. Hodges $^{4} \cdot$ Amy M. Taylor $^{1}$. \\ Gregory Bova ${ }^{5}$. Javad R. Azadi ${ }^{1}$
}

Received: 25 November 2020 / Revised: 16 February 2021 / Accepted: 25 February 2021 / Published online: 27 March 2021

(c) The Author(s), under exclusive licence to Springer Science+Business Media, LLC, part of Springer Nature 2021

\begin{abstract}
No guidance exists on how to safely perform modified barium swallows (MBS) in the midst of the COVID-19 pandemic or other communicable airborne respiratory infections (C-ARI). MBS has the potential to become an aerosol generating procedure (AGP) as it may trigger a cough or necessitate suctioning which may result in transmission of C-ARI putting patients and health care workers at risk. Regulations and best practices from international and US governmental and commercial agencies were reviewed. This review led to the multidisciplinary development of best practices of the safety measures and structural requirements to avoid transmission of SARS-CoV-2 or other C-ARIs when performing MBS. Implementation of these best practices resulted in structural changes to the fluoroscopy suite and protocol workflows. This enabled patients with COVID-19 to undergo MBS while maintaining patient and staff safety including mitigation of potential risk of onward transmission of SARS-CoV-2 to other patients. With proper modifications, MBS can be safely performed on patients with C-ARI such as COVID-19 while maintaining patient and health care worker (HCW) safety.
\end{abstract}

Keywords Modified barium swallow $\cdot$ Fluoroscopy $\cdot$ COVID-19 $\cdot$ SARS-CoV-2 $\cdot$ Airborne respiratory illnesses

\begin{tabular}{llll}
\multicolumn{2}{l}{ Abbreviations } & HCW & Health care worker \\
MBS & Modified barium swallows & WHO & World Health Organization \\
SARS-CoV-2 & Severe acute respiratory syndrome corona- & CDC & Centers for disease control \\
& virus 2 & PUI & Patients under investigation \\
COVID-19 & Coronavirus disease 2019 & ACH & Air exchanges per hour \\
C-ARI & Communicable airborne respiratory & CFM & Cubic feet per minute \\
& infections & HEPA & High-efficiency particulate air \\
AGP & Aerosol generating procedure & PPE & Personal protective equipment
\end{tabular}

ARI

Airborne respiratory illnesses

Alice R. Goldman

Alice.goldman@jhmi.edu

Jay K. Pahade

jay.pahade@yale.edu

Nicole A. Langton-Frost

Nlangto1@jh.edu

Chiquacta A. Hodges

chodges8@jhmi.edu

Amy M. Taylor

ataylo99@jhmi.edu

Gregory Bova

gbova1@jhmi.edu

Javad R. Azadi

jazadi1@jhmi.edu
1 Russell H. Morgan Department of Radiology and Radiologic Sciences, Johns Hopkins University School of Medicine, 1800 Orleans St, Zayed 4030B, Baltimore, MD 21287, USA

2 Yale Department of Radiology and Biomedical Imaging, Yale University School of Medicine, 333 Cedar St., New Haven, CT 06510, USA

3 Speech-Language Pathology, Department of Physical Medicine and Rehabilitation, Johns Hopkins Hospital, 600 North Wolfe Street, Osler 425, Baltimore, MD 21287, USA

4 Hospital Epidemiology and Infection Control, Johns Hopkins Hospital, 600 North Wolfe Street, Baltimore, MD 21287, USA

5 Department of Commissioning, Johns Hopkins Health System, 600 North Wolfe Street, Phipps Basement, Baltimore, MD 21287, USA 
HEIC Hospital epidemiology and infection control

HFFU

HEPA filter fan unit and exhaust configuration

\section{Introduction}

No guidance exists on how to safely perform modified barium swallows (MBS) in the midst of the COVID-19 pandemic or in patients with other airborne respiratory infections (ARI) such as tuberculosis or measles.

We report our experience creating a safe workspace for MBS in the setting of the COVID-19 pandemic at a single tertiary academic medical center. We found no existing policies or guidelines for performing this procedure on patients with COVID-19 or any other C-ARI. Our primary goal was to ensure the safety of staff and patients given current knowledge of SARS-CoV-2 transmission without impeding timely patient access to MBS. We also strove to limit onward transmission from asymptomatic patients who may be unknowingly infected and thus contagious with COVID-19 as they would normally have no special precautions.

\section{Background}

\section{What are the safety concerns?}

The World Health Organization (WHO) hypothesizes that "AGPs expose health care workers (HCWs) to respiratory pathogens, thereby increasing their risk of contracting these infectious diseases." [1]. AGPs are a cause for concern for HCWs as they may irritate the patient's airway causing them to cough or mechanically expel respiratory secretions into the air. These aerosols may travel and/or remain suspended in the air for an extended period of time. In the setting of C-ARIs like tuberculosis and measles with proven airborne transmission, negative pressure room and respirator use are considered the standard of care to mitigate the risk of disease spread. To our knowledge negative pressure rooms have not been routinely incorporated into diagnostic radiology fluoroscopy suites, potentially putting both patients and HCWs at increased risk when patients with C-ARI are examined.

During an MBS, patients are challenged with varying oral consistencies to assess oropharyngeal function, especially for evidence of laryngeal penetration and aspiration. An aspiration event may trigger a cough reflex which may necessitate suctioning if the aspirated bolus is not adequately cleared from the larynx. The MBS is considered an AGP by the Radiology Society of North America (RSNA), Society of Interventional Radiology (SIR), the United States' Centers for Disease Control (CDC), WHO, Dysphagia Research
Society (DRS) and American Speech-Language-Hearing Association (ASHA) [1-6]. Open airway suctioning is included on the CDC list of commonly considered AGPs as it can generate higher concentrations of infectious respiratory aerosols than coughing or sneezing alone [4]. The CDC acknowledges that stratifying the risk severity of different AGPs is challenging [4].

\section{Which patients are considered to be infected with SARS-CoV-2/COVID-19?}

Appropriate triaging of patients at risk for, contagious with, and recovered from COVID-19 is challenging given varying performance of current PCR tests available [7]. Reports have shown $21 \%$ of patients with 2 consecutive negative PCR tests subsequently had positive PCR so institutions have to account for the possibility of false negative results in patients with active infection [8].

Patients with positive SARS-Cov-2-PCR testing via nasopharyngeal swab are considered to have COVID-19 at our institution whether they are acutely symptomatic or positive but asymptomatic. Determining how to handle patients with prolonged PCR positive results weeks after onset of symptoms is challenging as it is unlikely that these patients remain contagious (classified as recovered from COVID19). Current data and CDC guidance suggest that the risk of a patient still being contagious is likely very small after 10 days for those with non-severe illness and after 20 days for those with severe (ICU level care) illness or a compromised immune system [9]. In patients who meet these conditions, COVID-19 precautions are removed and the patients are treated as recovered and no longer contagious.

At our institution, patients under investigation (PUI) do not undergo MBS because test results are available in under $24 \mathrm{~h}$, and MBS is considered a non-emergent procedure. If the patient's PCR test result is positive and the patient is deemed to have an active SARS-CoV-2 infection, MBS is performed in a negative pressure fluoroscopy suite when clinically appropriate and the patient is able to participate. These MBS requests are often made before the patient would be deemed noninfectious. For patients recently recovered we take the added precaution, when possible, of performing MBS at the end of the day to minimize the potential risks of onward transmission. For institutions without negative pressure facilities, MBS should ideally be deferred as clinically feasible until the patient is out of the contagious window.

\section{Safety in the fluoroscopy suite in the setting of ARI}

Fluoroscopy suites in Diagnostic Radiology are required to have at least 6 air exchanges per hour (ACH) for existing facilities with air exhausted directly to the outside or recirculated through appropriate filtration [10]. The formula 
for calculating $\mathrm{ACH}$ is $((\mathrm{CFM} \times 60 \mathrm{~min}) /$ volume of room $)$ where CFM equals the velocity of air flowing into or out of a space measured in cubic feet per minute (CFM). Using the $\mathrm{CDC}$ airborne contaminant removal table (Table 1) one can determine the necessary room closure time for desired contaminant removal based on the measured $\mathrm{ACH}$.

The direction air flows when the room door is open determines whether it has positive or negative pressure. Outside air flows into a negative pressure room which helps keep airborne pathogens within the room protecting patients and staff from airborne contamination. Positive pressure rooms maintain higher pressure inside the room so air preferentially flows out from the room protecting vulnerable patients from airborne infections and disease.

\section{Current recommended safety precautions for C-ARIs and patients with COVID-19}

Airborne precautions are recommended for C-ARI that remain suspended in the air over long distances [12, 13]. Patients with airborne infections such as measles, chickenpox, and tuberculosis should be treated in negative pressure rooms with the room air either exhausted directly to the outside or recirculated through high-efficiency particulate air (HEPA) filtration before return [12].

The CDC specifically states AGPs including open airway suctioning patients should be performed in a negative pressure room [4, 14]. While our fluoroscopy suites have wall suction and open airway suctioning is occasionally performed during MBS evaluations, we recognize that not all fluoroscopy suites have this available as a potential source of aerosolization. Our institution requires room closure of 45-60 min after a patient with COVID-19 undergoes any AGP. Both the CDC and WHO recommend airborne precautions for these patients undergoing any AGP [13]; all involved HCW should wear appropriate personal protective

Table 1 ACH values and time required for 99-99.9\% airborne contaminant removal. Adapted from the CDC airborne contaminant removal table [11]

\begin{tabular}{lll}
\hline $\begin{array}{l}\text { Airborne contaminant removal } \\
\text { Air changes/hour (ACH)-minutes to achieve desired \% airborne } \\
\text { contaminant removal }\end{array}$ \\
\hline ACH & $99 \%(\min )$ & $99.9 \%(\mathrm{~min})$ \\
\hline 6 & 46 & 69 \\
12 & 23 & 35 \\
15 & 18 & 28 \\
20 & 14 & 21 \\
50 & 6 & 8 \\
\hline
\end{tabular}

This is utilized to determine the duration of room closure for a fluoroscopy suite following performance of an AGP in a patient with a C-ARI equipment (PPE) including an N95 or equivalent respirator, eye protection, gloves, and a gown.

AGPs in patients with C-ARI should be performed by the most skilled operator available and only undertaken when absolutely necessary [15]. Efforts should be made to avoid unnecessary or potentially unsuccessful AGPs and minimize staff to only those providing direct patient care to reduce staff exposure and preserve PPE. A terminal clean is performed after completion of the procedure. Extra attention is given to general infection control techniques including clean-to-dirty workflow, proper donning and doffing of PPE, and hand washing. Patients remain masked until procedure begins and re-don their mask as soon as appropriate.

\section{Assessing the issues}

\section{Multidisciplinary team created}

We assembled a multidisciplinary team comprised of HCWs, facility workers, hospital epidemiology and infection control (HEIC) experts, and other stakeholders in our hospital to determine the best practices for safely performing MBS in patients with SARS-CoV-2 during the current COVID-19 pandemic.

\section{Infection control risk assessment performed}

All fluoroscopy suites should undergo an infection control risk assessment to identify and address potential risks related to MBS for the specific environment [16]. Negative pressure rooms protect those outside the room. As part of the assessment, one must determine whether the fluoroscopy suite air is recirculated or not; exposure to contaminated recirculated air poses potential risks to patients and staff. We recommend each institution explore whether converting their fluoroscopy suite to negative pressure is feasible given concern for potentially contaminated air recirculating to nearby radiography/ fluoroscopy rooms, waiting areas, and staff work areas. CDC guidance states that negative pressure rooms should be used (ideally) for all patients undergoing AGPs with confirmed and suspected COVID-19 infection as well as all C-ARI. It is critical to note that relying only on the recommended air exchanges based on CDC table (Fig. 1) does not address the potential risk of exposure to contaminated recirculated air.

\section{Fluoroscopy suite assessment performed}

All of our existing fluoroscopy suites for MBS studies were positive pressure and were not vented to the outside so we determined negative pressure conversion would be necessary. Based on CDC guidelines for our fluoroscopy suite with pre-conversion $\mathrm{ACH}$ value 12.5 , room closure times 
Fig. 1 Facilities drawing for fluoroscopy suite conversion. This demonstrates the final layout for a converted fluoroscopy suite including the doffing area in an adjacent hallway

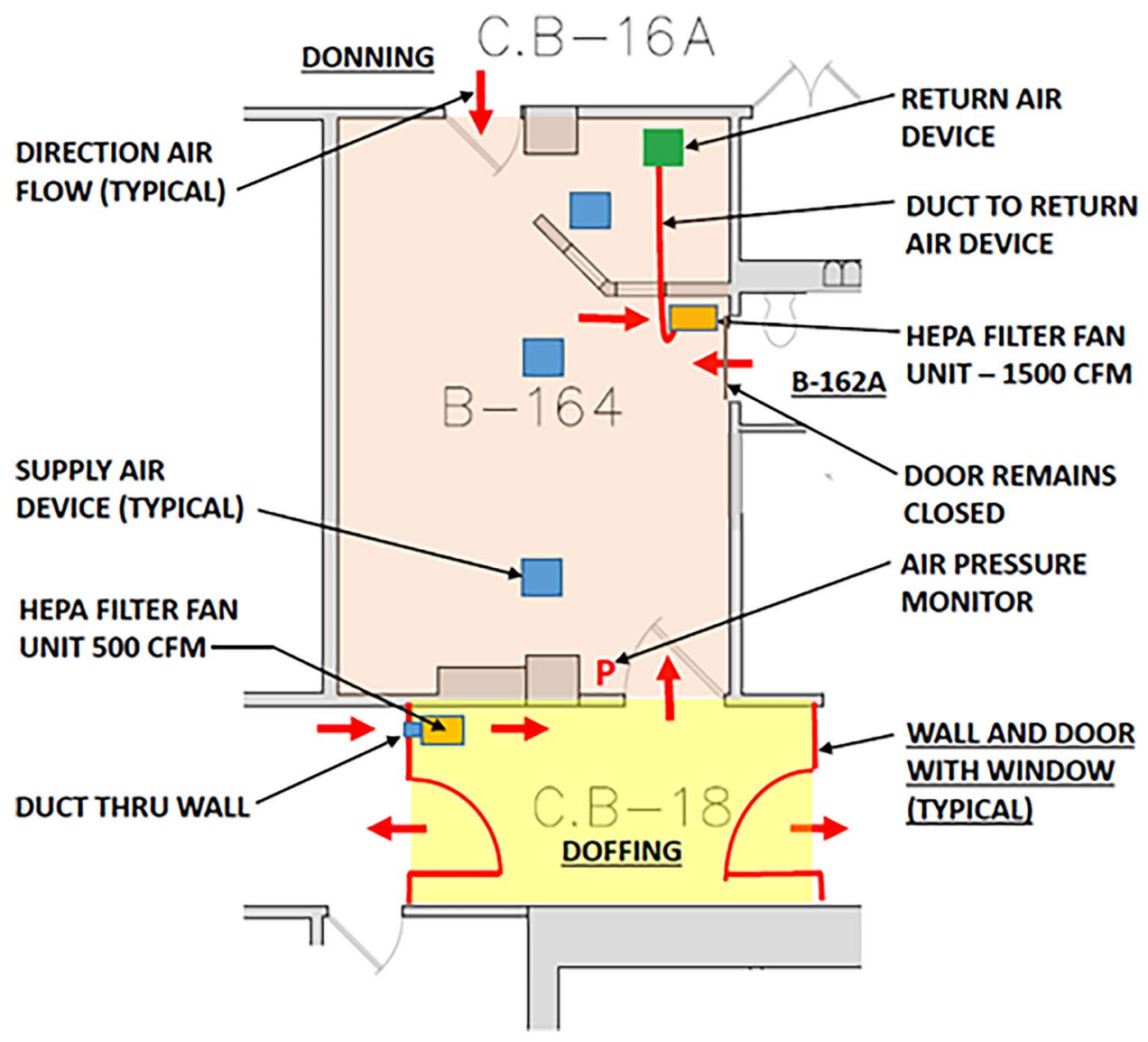

of 28 and $41 \mathrm{~min}$, respectively, would be needed to achieve 99\% and $99.9 \%$ airborne contaminant removal (Fig. 1).

\section{Actions taken}

\section{Fluoroscopy suite converted to negative pressure}

The facility's staff used the air handling capability and room layout to determine the appropriate HEPA filter fan unit and exhaust configuration (HFFU) needed to convert the room to negative pressure. The HFFU filters the air before returning it to the air handling units after which the previously contaminated air can be safely recirculated. Pressure gauges were installed to enable onsite confirmation that negative pressure had been achieved.

An additional positive pressure HEPA-filtered (separate from the suite itself) ante-room was created by closing off a segment of the adjacent hallway by adding walls with doors to enable safe patient and staff entry/exit as well as to provide space for safe donning and doffing of PPE (Fig. 1). There is an alternate hallway available for passage when the doors are closed. At the lead author's institution, total conversion was successfully performed over 2-3 days at a cost of approximately $\$ 7000$.

\section{Options for facilities unable to convert to negative pressure}

If the patient is housed in a negative pressure room, performing the MBS at the patient's bedside would eliminate the need to transport a patient with an ARI but would require a portable $\mathrm{C}$-arm fluoroscopy unit $(\mathrm{C}$-arm) that could fit into the patient's room and record video (needed for MBS), such as a vascular C-arm. Assessment of any available negative pressure rooms in the radiology department should also be performed. For example, at the lead author's institution, the pediatric fluoroscopy suite was negative pressure. However, the small field of view on the system precluded diagnostic MBS performance in seated adult patients. If there are no available options and full negative pressure conversion of the fluoroscopy suite is not possible, placing a HEPA filter or creating a doffing area with HEPA filtration may help mitigate the risk by increasing air exchange rates. 


\section{Determine room closure times for MBS during the COVID-19 pandemic}

Safe performance of MBS during the current pandemic requires appropriate room closure times and appropriate use of PPE during the procedure.

We developed a flow map (Fig. 2) stratifying patients into 2 groups to determine optimal room closure time: group I are those with known active or recovered SARS-CoV-2 infection or PUI and group II are those untested or with a negative test. This latter group includes those with negative SARS-CoV-2-PCR test within 48-72 h, those tested beyond prescribed time, and those who have not been tested but are asymptomatic (non-PUI). Although treating all patients as if they had COVID-19 would eliminate variation in our practice, instituting a 45-60-min room closure after every MBS would markedly reduce the scheduling capacity of the fluoroscopy suite. Given the high demand for MBS exams, we further stratified our approach to room closure times based on whether or not an event occurred during the procedure that could potentially generate a significant amount of aerosol.

For group II patients who cough or require airway suctioning during MBS (and could be asymptomatic but infected), we used the CDC table to determine evidence based appropriate room closure time as these patients do not have to follow the hospital mandate of 45 -60 min used for group I patients. This approach allows us to mitigate the risk of onward transmission from potentially infected patients to the next patient coming for MBS which requires them to remove their mask.

The room closure time should be based on the measured $\mathrm{ACH}$ value of the fluoroscopy suite. For the lead authors, the renovated negative pressure fluoroscopy suite had an $\mathrm{ACH}$ of 27. The suggested time needed to clear $99.9 \%$ of aerosol at this ACH per CDC guidelines (Table 1) is $21 \mathrm{~min}$. This was deemed to also be sufficient for negative or untested patients (group II) in whom an aerosol inducing event occurs. For institutions unable to convert to negative pressure, a room closure time of 46-69 min would be necessary to achieve 99-99.9\% clearance based on the minimum expected $\mathrm{ACH}$ for a radiography room $(\mathrm{ACH}$ of 6) (Table 1), if an aerosol inducing event occurred. If no aerosol inducing event occurs, there is no prescribed room closure time but we do keep the suite's doors closed in between patients to further optimize room air safety. We perform standard or terminal room cleaning appropriate to each patient which creates a 5-10-min room turnover time.

For patients with active infection, full airborne precaution PPE is utilized. For other patients, we still use N95 masks/PAPR, face shields, and gloves, but we omit the protective gown used in full airborne precaution due conservation efforts to ensure gown availability for other patient encounters. For all patients undergoing MBS in a room without negative pressure, full airborne precaution PPE (N95/PAPR, eye protection, gown, and gloves) is advised, since one cannot predict which procedure will become an AGP. We currently reuse N95 masks and eye protection until visibly damaged, soiled, or with altered fit which has allowed us to conserve supplies during periods of low availability but still protect our staff.

\section{Created an algorithm to avoid unsuccessful MBS attempts}

We developed an algorithm (Fig. 3) in an effort to optimize scheduling of patients for MBS, balancing the need for timely results with the likelihood of achieving a successful exam. Primary goals include not performing MBS on patients with cognitive or physical limitations which would preclude their essential participation in the study and optimizing timing such that MBS results will immediately inform a pending management decision or plan which will impact clinical care.
Fig. 2 Risk stratification for prescribed room closure for patients undergoing MBS. This determination is made based on whether a patient has a confirmed C-ARI and whether or not an AGP occurred

\section{Patient stratification for post-MBS room closure times}

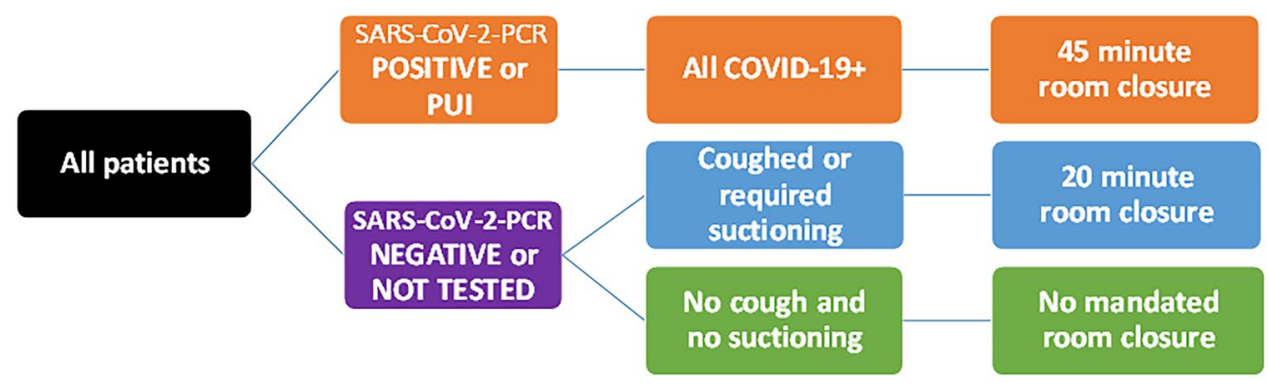




\section{Algorithm to balance patient readiness for MBS and appropriate timing of MBS}
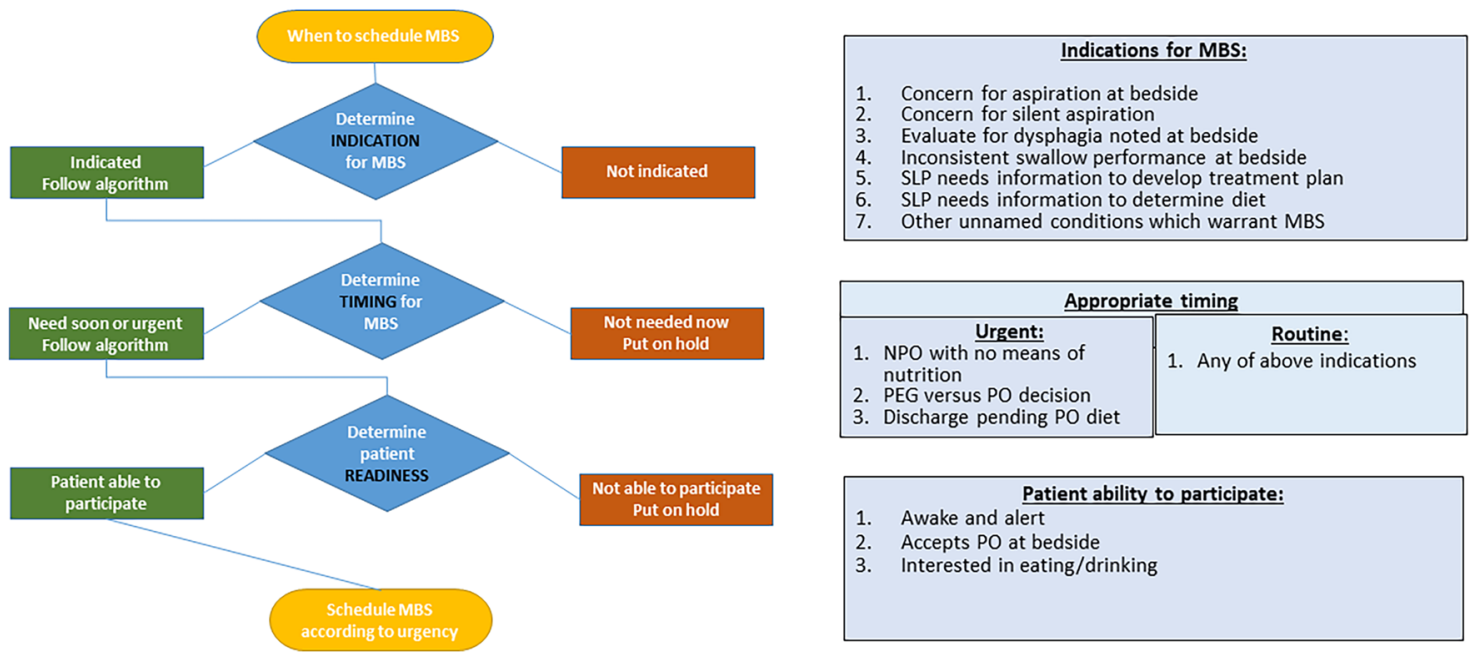

Fig. 3 Algorithm to optimize scheduling of MBS by balancing patient readiness for MBS and timely scheduling of MBS. In an effort to avoid a "wasted" time slot for MBS, we assess patient readiness and try to schedule the MBS at a time when it will directly impact patient management

\section{Additional considerations}

\section{Is onward transmission of SARS-CoV-2 a risk of MBS?}

Onward transmission is the transmission of a communicable ARI like SARS-CoV-2 from an asymptomatic infected patient to another patient subsequently undergoing MBS. This is a concern given potential aerosol generation during an MBS and that patients are unmasked throughout the procedure. Recent data suggest a substantial percentage of onward transmission occurs during the pre-symptomatic phase of infection or from patients who remain asymptomatic throughout the course of their infection [14]. Instances of MBS being performed on patients who were not known to have SARS-CoV-2 because they were asymptomatic or were pre-symptomatic has occurred at our institutions. This means the patient completed the MBS during a potential period of high viral load and high infectivity.

\section{Can universal SARS-CoV-2 PCR testing prevent onward transmission?}

While universal PCR testing is ideal, it has not been feasible at our institutions for every outpatient MBS. Many radiology departments may not have the necessary infrastructure in place to allow for pre-procedure test ordering and follow up of results. Asking ordering providers to take on this task also increases their workload and has to be communicated by radiology staff or electronically at time of order entry. In addition, PCR tests have variable sensitivity and specificity values with potential of false negative results based on test performance and adequacy of sample collected. The CDC states a negative SARS-CoV-2-PCR test means "most likely you do not currently have active COVID-19 infection"[17]. A May 2020 article quantified the risk of false negative SARS-CoV-2 PCR testing based on time of testing. The lowest reported false negative rate was $21 \%$ one week following exposure, with rates as high as $67-100 \%$ up to four days preceding symptom onset and $38 \%$ on the first day of symptoms [18]. False negatives pose a health threat as a patient with a false negative test is infected with SARS-CoV-2, but does not know it. Therefore, special considerations must be given when SARS-CoV-2 PCR test results are the "basis for removing precautions intended to prevent onward transmission" [18]. While PCR testing for SARS-CoV-2 has become more widely available over time, our institutions have not opted to mandate pre-procedure testing before MBS. By following the flow chart (Fig. 2) regarding room closure, we mitigate risk of harm that could occur if aerosol generation occurs in a patient who has a false negative test result while providing the needed clinical care.

\section{Summary}

Through our multidisciplinary approach and utilizing current national infection control recommendations, we successfully adopted safety precautions regarding PPE, room closure times, and converted our fluoroscopy suite to a negative pressure HEPA-filtered environment. We implemented room closure practices to address the risk of onward transmission of ARI from patients untested or negative for 
SARS-CoV-2. We describe potential approaches for facilities unable to make the conversion. Furthermore, we created an algorithm with speech language pathologists to optimize MBS patient scheduling in an effort to decrease unsuccessful MBS attempts.

\section{Response to implemented changes}

The conversion to the negative pressure HEPA-filtered fluoroscopy suite has been well received especially given the surge in cases. HCWs have described feeling relief in knowing they are in a safer environment evaluating and treating patients with COVID-19 who may cough or require suctioning, and they can reassure these patients they are in a safe environment. The only criticism has been that the HEPA filter unit is noisy, which can make it more difficult to communicate with patients, especially when wearing masks. After completion of construction, there has been no impact on workflow or schedule capacity. Facility maintenance staff will need to periodically service and audit the equipment to ensure safe operation.

The scheduling algorithm we developed has resulted in fewer unsuccessful studies as greater attention is paid to balancing the urgency of the exam with the patient's ability to successfully participate in the MBS. By considering both factors, we have reduced the number of unsuccessful studies.

During the first 3 months following the room conversion, 31 patients with active or resolved SARS-CoV-2 underwent MBS in our negative pressure room. An additional 2 patients with tuberculosis have also undergone MBS in our negative pressure room during this period.

Particular strengths of our approach include the multidisciplinary approach to mitigating risks in the setting of a pandemic which resulted in a relatively low-cost immediate solution with long term benefits. Management of infection control during MBS in patients with communicable ARI prior to COVID-19 had not been adequately addressed; this solution provides a safe environment for future patients with C-ARI such as tuberculosis. This paper also reviews factors to be addressed to limit the risk of onward transmission of SARS-CoV-2 infection. We recommend institutions interested in performing fluoroscopic AGPs on patients with communicable ARIs such as SARS-CoV-2 to explore the possibility of room conversion to negative pressure. Our findings are generalizable and can be adopted by other institutions addressing the immediate need for a safe environment to assess patients with COVID-19 but also for the ongoing need to address swallowing issues in patients with other C-ARIs. For facilities unable to convert to a negative pressure room, an infection control assessment of the suite is recommended including $\mathrm{ACH}$ determination and determining room ventilation type since recirculated air may pose safety hazards. Information necessary to assess the safety of facilities and some alternative solutions for facilities unable to make the conversion to negative pressure HEPA filtration is provided.

\section{Conclusion}

Through modifications to the fluoroscopy suite and appropriate triaging, MBS can be performed on patients with SARSCoV-2 and other communicable airborne respiratory infections to increase health care worker and patient safety.

Author contributions Dr. Goldman substantially contributed to the conception and design of the work, acquisition and interpretation of the relevant information, the drafting and revision of the manuscript, and approved the final version of the manuscript. Dr. Pahade substantially contributed to the acquisition and interpretation of the relevant information, revision of the manuscript, and approved the final version of the manuscript. Ms. Langton substantially contributed to the design of the work, acquisition and interpretation of the relevant information, revision of the manuscript, and approved the final version of the manuscript. Mr. Bova substantially contributed to the design of the work, acquisition and interpretation of the relevant information, revision of the manuscript, and approved the final version of the manuscript. Ms. Hodges contributed to the design of the work, acquisition and interpretation of the relevant information, revision of the manuscript, and approved the final version of the manuscript. Ms. Taylor substantially contributed to the design of the work, acquisition and interpretation of the relevant information, revision of the manuscript, and approved the final version of the manuscript. Dr. Azadi contributed to the design of the work, acquisition and interpretation of the relevant information, revision of the manuscript, and approved the final version of the manuscript. All authors agree to be accountable for all aspects of the work, including ensuring that questions related to the accuracy or integrity of any part of the work are appropriately investigated and resolved.

Data availability The authors declare that they had full access to all of the data in this study and the authors take complete responsibility for the integrity of the data and the accuracy of the data analysis.

\section{Compliance with ethical standards}

Conflict of interest Jay K Pahade, MD—Consultant GE healthcare. All other authors declare no conflict of interest.

\section{References}

1. World Health Organization (2007) Infection prevention and control of epidemic- and pandemic-prone acute respiratory diseases in health care. https://apps.who.int/iris/bitstream/handle/10665/ 69707/WHO_CDS_EPR_2007.6_eng.pdf;jsessionid=1D73D 5EA9CE322D9D12C844B51CFC592? sequence $=1$. Accessed 20 Aug 2020

2. Mossa-Basha M, Azadi J, Ko J, Klein J, Meltzer C, Force C-T (2020) RSNA COVID-19 Task Force: Best Practices for Radiology Departments during COVID-19. http://Rsna.org/COVID-19. Accessed 20 Aug 2020

3. Society of Interventional Radiology (2020) Aerosol Generating Procedures Performed by Interventional Radiology Clinical 
Notification from the Society of Interventional Radiology. https:// www.sirweb.org/practice-resources/covid-19-resources/covid-19clinical-notification-3-26-20/. . Accessed March 2020

4. Center for Disease Control and Prevention (2020) Clinical Questions about COVID-19: Questions and Answers. https://www. cdc.gov/coronavirus/2019-ncov/hcp/infection-control-faq.html. Accessed 20 Aug 2020

5. Dysphagia Research Society (2020) COVID-19 Information and Resources: Risk Management of AGPs for Dysphagia Care. https://www. dysphagiaresearch.org/page/COVID19AGPs. Accessed 20 Aug 2020

6. American Speech-Language-Hearing Association (ASHA) (2020) ASHA Guidance to SLPs Regarding Aerosol Generating Procedures. https://www.asha.org/SLP/healthcare/ASHA-Guidance-toSLPs-Regarding-Aerosol-Generating-Procedures/. Accessed 20 Aug 2020

7. He J-L, Luo L, Luo Z-D, Lyu J-X, Ng M-Y, Shen X-P, Wen Z (2020) Diagnostic performance between CT and initial realtime RT-PCR for clinically suspected 2019 coronavirus disease (COVID-19) patients outside Wuhan, China. Respir Med 168:105980-105980. doi:https://doi.org/10.1016/j.rmed.2020. 105980

8. Xiao AT, Tong YX, Zhang S (2020) False negative of RT-PCR and prolonged nucleic acid conversion in COVID-19: Rather than recurrence. J Med Virol. doi:https://doi.org/10.1002/jmv.25855

9. Center for Disease Control and Prevention (2020) Duration of Isolation and Precautions for Adults with COVID-19. https:// www.cdc.gov/coronavirus/2019-ncov/hcp/duration-isolation.html. Accessed 19 Oct 2020

10. American Society of Heating Refrigerating and Air-Conditioning Engineers Inc. (2017) ANSI/ASHRAE/ASHE Standard 1702017. Ventilation Of Health Care Facilities.

11. Center for Disease Control and Prevention (2020) Table B.1. Air changes/hour $(\mathrm{ACH})$ and time required for airborne-contaminant removal by efficiency $*$.
12. Center for Disease Control and Prevention (2007) Precautions to Prevent Transmission of Infectious Agents Guideline for Isolation Precautions: Preventing Transmission of Infectious Agents in Healthcare Settings. https://www.cdc.gov/infectioncontrol/guide lines/isolation/precautions.html. Accessed 20 Aug 2020

13. World Health Organization (2020) Infection prevention and control. https://www.who.int/csr/disease/coronavirus_infections/preve ntion_control/en/. Accessed 20 Aug 2020

14. Center for Disease Control and Prevention (2020) Interim Clinical Guidance for Management of Patients with Confirmed Coronavirus Disease (COVID-19). https://www.cdc.gov/coronavirus/2019ncov/hcp/clinical-guidance-management-patients.html. Accessed 20 Aug 2020

15. Center for Disease Control and Prevention (2020) Interim Infection Prevention and Control Recommendations for Healthcare Personnel During the Coronavirus Disease 2019 (COVID-19) Pandemic. https://www.cdc.gov/coronavirus/2019-ncov/hcp/infec tion-control-recommendations.html. Accessed 20 Aug 2020

16. Center for Disease Control and Prevention (2019) Infection Control Assessment Tools. https://www.cdc.gov/hai/prevent/infectioncontrol-assessment-tools.html. Accessed 20 Aug 2020

17. Center for Disease Control and Prevention (2020) Test for Current Infection. https://www.cdc.gov/coronavirus/2019-ncov/testi ng/diagnostic-testing.html. Accessed 20 Aug 2020

18. Kucirka LM, Lauer SA, Laeyendecker O, Boon D, Lessler J (2020) Variation in False-Negative Rate of Reverse Transcriptase Polymerase Chain Reaction-Based SARS-CoV-2 Tests by Time Since Exposure. Ann Intern Med 173 (4):262-267. doi:https://doi. org/10.7326/M20-1495

Publisher's Note Springer Nature remains neutral with regard to jurisdictional claims in published maps and institutional affiliations. 